\title{
The Role of a Handheld Ultrasound Device to Facilitate Remote Learning for a Point-of-Care Ultrasound Certificate Program
}

\author{
Alok Moharir ${ }^{1,2}$ \\ Joseph D Tobias (iD) ${ }^{1,2}$ \\ 'Department of Anesthesiology \& Pain \\ Medicine, Nationwide Children's \\ Hospital, Columbus, OH, USA; \\ ${ }^{2}$ Department of Anesthesiology \& Pain \\ Medicine, The Ohio State University \\ College of Medicine, Columbus, \\ $\mathrm{OH}$, USA
}

\begin{abstract}
Point-of-care ultrasound is a growing field within anesthesiology as well as several other medical and surgical specialties. The recent development of handheld ultrasound devices has the potential to expand the use or this technology by decreasing the cost and increasing accessibility for the healthcare provider. These handheld devices may be used to assist with education, training, and even direct patient care. We outline our process for using a handheld ultrasound device, the Lumify, to assist in the completion of a point-of-care ultrasound certificate program offered by the American Society of Anesthesiologists. We outline the unique advantages of this device as it relates to cost, portability, and applicability of its web-based technology compared to a traditional standalone ultrasound machine for the completion of this course.
\end{abstract}

Keywords: ultrasound, point-of-care ultrasound, POCUS, hand-held

\section{Introduction}

Point-of-care ultrasound (POCUS) is now recognized as a core competency within anesthesiology. ${ }^{1-3}$ Advantages include its speed, portability, non-invasive nature, avoidance of radiation, and the ability to provide results in real time at the bedside. ${ }^{4-6}$ Despite these advantages, barriers to its full integration and implementation into clinical practice may still exist. Within each department, there may be only a limited number of faculty who are proficient in all aspects of POCUS. While ultrasound guided procedures have become commonplace in anesthesia practice for vascular access and regional anesthesia using primarily a linear probe, diagnostic POCUS requires additional training and expertise. Additionally, these procedures require the use of multiple types of probes to ensure image acquisition. In particular, a phased array probe is necessary for cardiac ultrasound while a curvilinear probe is helpful for gastric and lung ultrasound. ${ }^{4,5}$ These technology needs may further increase the cost and equipment barriers for training and implementation into routine clinical practice.

While the standard for the practice of POCUS in anesthesiology has generally included larger, stand-alone devices such as the Sonosite SII or GE Venue 50, the development of handheld devices has the potential to expand POCUS training by making ultrasound technology more affordable, more portable, and more accessible to the anesthesiologist in various clinical scenarios and situations. Many of these novel, hand-held devices are compatible with pre-existing smartphones and tablets,
Correspondence: Alok Moharir Department of Anesthesiology \& Pain Medicine, Nationwide Children's Hospital, 700 Children's Drive, Columbus, $\mathrm{OH}$ 43205, USA

Tel + I 614 722-4200

Fax + $614722-4203$

Email Alok.

Moharir@Nationwidechildrens.org 
thereby improving imaging acquisition and viewing. They have a high degree of diagnostic accuracy at a fraction of the cost of the stand-alone ultrasound machines. ${ }^{7}$ Many of these devices can also use cloud-based technology to capture, store, and share images in a Health Insurance Portability and Accountability Act (HIPAA) compliant manner. Hand-held ultrasound devices can also be used to facilitate and enhance remote learning by providing a pathway for practicing anesthesiologists to submit ultrasound images to outside faculty mentors for review if their own department lacks sufficient expertise. ${ }^{7}$ These remote learning opportunities refer to the use of virtual learning or connection with mentors through the internet and other virtual platforms. This allows teaching by experts when there may not such expertise available at one's home institution.

\section{American Society of Anesthesiologists' POCUS Course}

The American Society of Anesthesiologists (ASA) has recently introduced a diagnostic point-of-care ultrasound course for physician anesthesiologists. This is a comprehensive program requiring proof of prior completion of an in-person POCUS training session, image interpretation through online case based modules, image acquisition training, and a final examination to test for knowledge. Successful fulfillment of these steps will result in a certificate of completion from the ASA. This course can be used to help validate anesthesiology departments for credentialing and granting of privileges in diagnostic point-of-care ultrasound within the hospital. Particular focus of this course is on gastric ultrasound for verification of nil per os (NPO) status, lung ultrasound to evaluate for pathology, and cardiac and IVC ultrasound for evaluation of function, pathology, and intravascular volume status.

For image acquisition training, physicians are required to upload a subset of ultrasound video clips in MPEG-4 (MP4) format for a faculty mentor to review. This includes scans of the gastric antrum in the supine and right lateral decubitus position, lung ultrasound in the anterior and posterolateral lung fields, and cardiac scans in the parasternal long axis, parasternal short axis, apical four chamber, subcostal four chamber, and inferior vena cava views. These clips can be reviewed by either a local mentor at one's institution or submitted online to be reviewed by an ASA faculty member if a local mentor is not available. Clips are removed of all personal health information (PHI) before being sent to a mentor in a HIPAA compliant manner. In order to ensure video quality, specific criteria must be met for each scan prior to uploading for review. This includes proper image orientation, depth, gain, inclusion of identifiable landmarks, and appropriate function according to criteria set by the ASA (ASA criteria for POCUS images). ${ }^{8}$

\section{Image Acquisition and Storage}

For image acquisition, we used a Philips Lumify (Philips Healthcare, Cambridge, MA) handheld ultrasound probe connected to a Samsung Galaxy tablet with the Lumify mobile app (Figure 1). A phased array probe (1-4 MHz) was used for the cardiac and inferior vena cava (IVC) scans, a curvilinear probe $(2-5 \mathrm{MHz})$ for the gastric and lung scans in the diaphragmatic region, and a linear probe (4-12 MHz) for the anterior lung fields. All videos met ASA standards for quality (Figures 2-4). Videos were saved to the Philips Lumify app as three second clips in a HIPAA compliant manner with all personal health information (PHI) removed (Supplementary Videos 1, $\underline{2}$ and $\underline{3}$ ). A secured, password protected Google Drive folder was created as an export destination for the videos. The images were saved in MP4 format as requested by the ASA POCUS committee (Figures 5 and 6). The files were then uploaded to the ASA website for review by a faculty mentor.

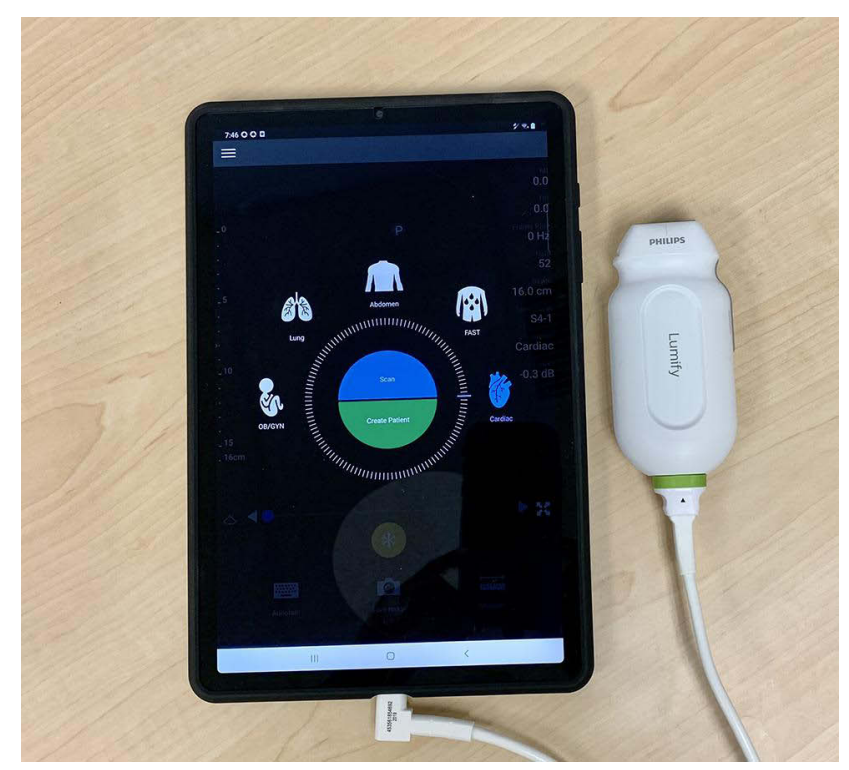

Figure I Philips Lumify S4-I broadband phased array probe connected to a Samsung Galaxy tablet with the Lumify app. 


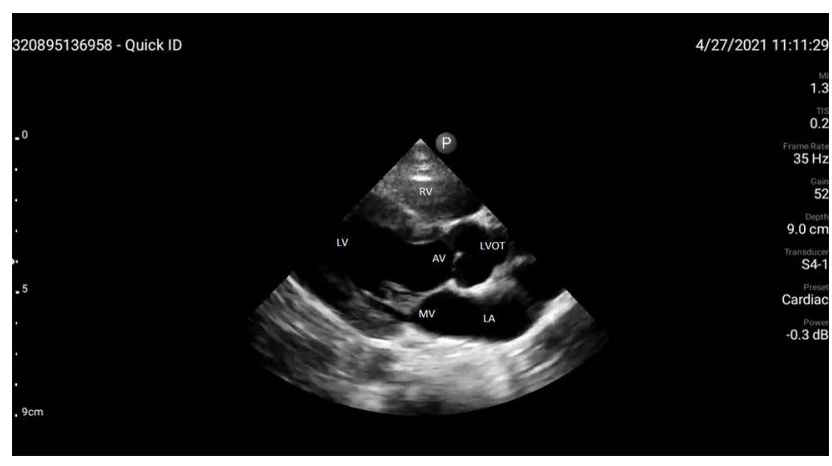

Figure 2 Cardiac ultrasound parasternal long axis view using a Philips Lumify S4I broadband phased array probe. This is a screenshot of a three second MP4 video clip. The MP4 clip is also available for viewing. The following structures are labelled.

Abbreviations: LV, Left Ventricle; LA, Left Atrium; LVOT, Left Ventricular Outflow Tract; RV, Right Ventricle; AV, Aortic Valve; MV, Mitral Valve.

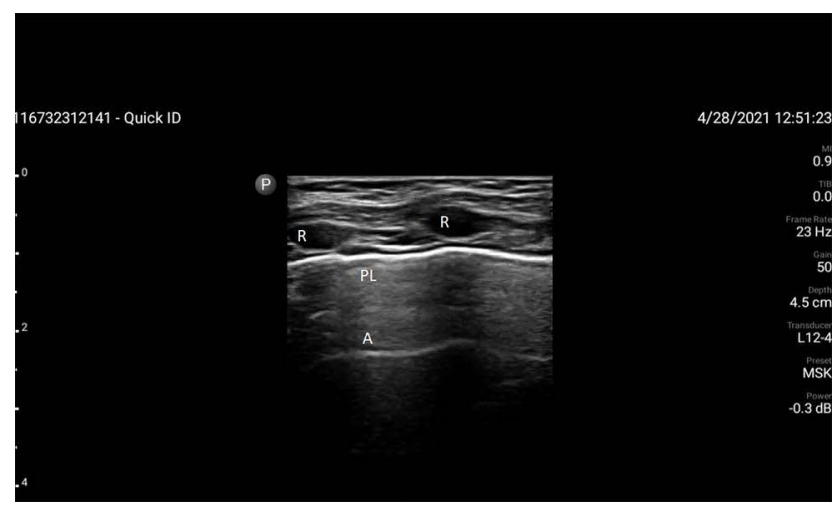

Figure 3 Lung ultrasound in the anterior lung field using a Philips Lumify LI2-4 broadband linear probe. This is a screenshot of a three second mp4 video clip. The MP4 clip is also available for viewing. The following structures are labelled.

Abbreviations: R, Ribs; PL, Pleural Line; A, A-Line, a horizontal artifact indicating a normal lung surface.

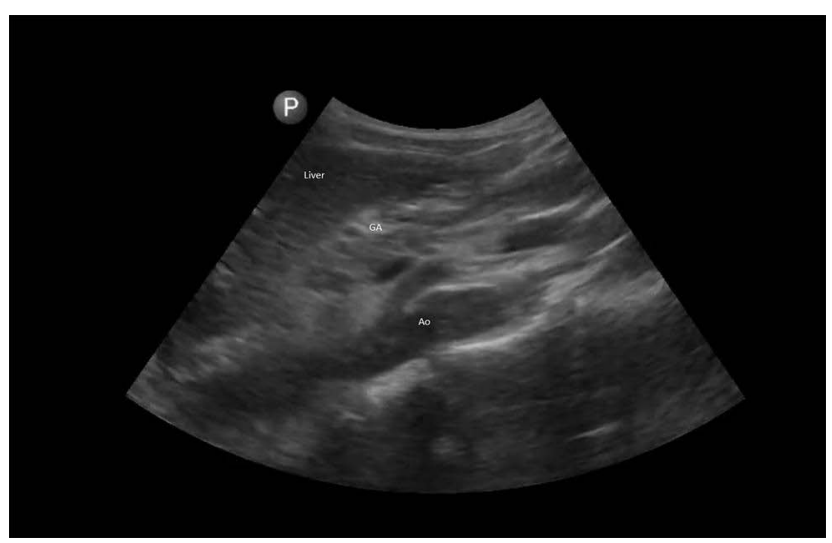

Figure 4 Gastric ultrasound in the right lateral decubitus (RLD) position using a Philips Lumify C5-2 broadband curvilinear probe. This is a screenshot of a three second mp4 video clip. The MP4 clip is also available for viewing. The liver; GA (gastric antrum), and Ao (Aorta) are labelled.
$2: 41000$

$\leftarrow$ Export Destinations + ADD NEW

Drive

Local Directory or Media raw:/storage/emulated/0/Download/_DriveSyncFiles Image Resolution 720p HD widescreen

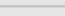

Figure 5 Google drive folder created as an export destination on the Philips Lumify app.

\section{Discussion}

Diagnostic POCUS is a growing field that involves multiple medical specialties. While other specialties such as Emergency Medicine and Intensive Care have long established POCUS clinical use and guidelines in their practice, POCUS use, training, and credentialling has recently become incorporated into Anesthesiology and its training. It is now also recognized as a core competency by the Accreditation Council in Graduate Medical Education (ACGME) for Anesthesiology. ${ }^{9}$ The training for anesthesiology residents, fellows, and faculty includes exposure to a combination of written materials, didactic lectures, hands-on teaching, case based modules, and image acquisition training.

We suggest that the incorporation of handheld ultrasound devices can greatly facilitate this training. These novel devices are more portable and less expensive than stand-alone ultrasound machines. ${ }^{10}$ In the anesthesiology arena, they can be used in remote or off-site areas such as radiology or procedural sedation centers when the larger stand-alone ultrasound machines may not be available due to space or cost constraints. Their portability allows them to be easily carried to these areas when needed. They offer greater flexibility in teaching and image acquisition since some ultrasound probes that are not as commonplace on ultrasound machines in anesthesiology departments, such as the phased array and curvilinear probes, can become more readily available. In addition, with the feasibility and easy access to websites, images used for educational purposes can be sent remotely to a POCUS mentor at an outside institution for review in a HIPAA compliant manner.

As these techniques are relatively novel in the field of anesthesiology, it will be necessary to train the current cohort of practicing anesthesiologists in addition to ensuring that our residents and fellows are trained in these techniques. This "Training the Trainer" has been suggested as a particular challenge in establishing use of point-ofcare ultrasound programs. ${ }^{10}$ By enhancing remote 


\section{3:46 \\ 4 Search \\ $<$ DriveSyncFiles}

Name $\uparrow$

0

image_305372756965.mp4

Modified Apr 28, 2021

( image_616129653044.mp4

Modified Apr 27, 2021

-

image_801066259536.mp4

Modified Apr 28, 2021
.1 LTE

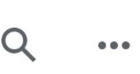

ㅍII

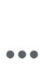

$\bullet \bullet$

$\bullet$

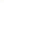

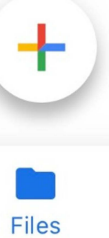

再

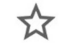

임.

Files

Figure 6 Saved images in the google drive folder. These images were then uploaded to the ASA website for review. All images were removed of personal health information and were stored in a HIPAA compliant manner.

learning, teaching can be concentrated and directed to more established POCUS experts. This process allows for the training of local experts without the meeting time and expense of attending courses away from one's home institution. This can not only help with initial POCUS certification, but can also aid in continuous quality improvement, which is crucial for POCUS programs.
There are currently various hand-held ultrasound devices that are practical for the practicing anesthesiologist. Our current preference for the Philips Lumify was based on several factors. First, it provides a high image quality. Clinical investigation has demonstrated superior image quality with the Lumify when compared with other handheld devices and showed accuracy equivalent to stand-alone ultrasound machines for specific, focused, POCUS functions. ${ }^{11,12}$ The Lumify probes have a similar look and feel to the standard ultrasound probes from the larger machines. Their size and footprint are also identical. The Lumify uses piezo-electric crystal technology similar to a traditional ultrasound. These factors may provide an easier transition from using the handheld device for teaching purposes to using a larger standalone machine inside the operating room setting.

The Philips Lumify has options for B-Mode, color Doppler mode, and M-Mode scanning. Most point-of-care functions in anesthesiology require B-mode for qualitative analysis of organ function. Color Doppler can assist with the evaluation of cardiac valvular function and during vascular access. M-Mode can be useful in lung ultrasound when identifying the seashore sign for the presence of lung ventilation and the barcode sign for the absence of lung ventilation. ${ }^{13-15}$ Unlike other handheld devices, the Lumify probe does not need to be charged separately. Only the smartphone or tablet used to display the images needs to be charged. Additionally, the device can be connected to either a smartphone (thereby increasing its portability) or a large tablet if needed to improve image analysis.

Finally, we appreciated the flexibility of having multiple export destinations for our saved images. In addition to storing images on the tablet, they could be emailed, stored in a local area network, or synced to a hospital's picture archiving and communication system (PACS); all in a secured, encrypted, HIPAA compliant manner. Users have complete control over how the images are managed. They could either be stored as still images or as video clips. Unlike other devices, a paid subscription is not required. However, users do have the option to upgrade to a paid service that allows one-on-one telehealth guidance with a healthcare professional in real time via the Reacts digital software platform (Innovative Imaging Technologies Inc, Montreal, Canada).

As handheld ultrasound technology becomes more ubiquitous and providers begin to use them more for direct patient care, it will be mandatory to work with the hospital's IS or IT department to ensure that images are saved, 
stored, and annotated in compliance with hospital policy. In the case of the Philips Lumify, a business agreement needs to be signed by hospital administration if images have the potential to contain personal health information. Ideally, a subset of these images should be periodically reviewed by members of the point-of-care ultrasound team for quality assurance.

Despite the many advantages of the Philips Lumify handheld ultrasound system, certain limitations exist. First, multiple types of Lumify probes are required depending on the depth and location of the desired scan. This is similar to a stand-alone ultrasound machine. Other hand-held devices, such as the Butterfly $\mathrm{iQ}$, have a microchip in place instead of a piezoelectric crystal that is capable of emulating multiple types of probes; thereby allowing the use of one probe for multiple functions. ${ }^{7}$ Each Lumify probe weighs less than five ounces and all three probes and a tablet easily fit into a shoulder bag to carry. Our impression was that the images of the Lumify device were more similar to a stand-alone ultrasound machine. However, as this technology is rapidly changing and there are many personal preferences that will go into the discussion process, we would recommend evaluating several of the hand-held devices before making a purchase to determine how best to balance cost, convenience, and comfort with image quality. The decision is made more difficult by the current lack of evidence-based medicine in our literature. A literature search of POCUS in anesthesiology reveals a paucity of clinical trials comparing hand-held ultrasound devices with each other and with stand-alone ultrasound machines. As this technology becomes more common in medical practice, it will be important to conduct rigorous studies showing the benefits and limitations of each device for image acquisition, training, education, and patient care.

Diagnostic POCUS is a growing field within the specialty of anesthesiology. Its utility in our practice has been recognized by incorporation of its training into residency and fellowship core competencies acceptance. As this practice continues to expand, it will be important to further establish guidelines for training, didactic and hands-on teaching, credentialling, and continuous quality improvement analysis. Individual departments will need to determine how best to incorporate training of their residents, fellows, and faculty. We believe that the ASA course can serve as a useful foundation for the development of these processes. They offer online lectures, case based modules, and image acquisition training with an ASA approved mentor. The development of hand-held ultrasound technology can further assist with this training. These hand-held devices are less expensive, more portable, and may be more accessible to the anesthesiologist than a stand-alone machine. They can also provide a high degree of diagnostic accuracy for specific, focused, pointof-care functions. In our experience, we have found that the unique features of the Philips Lumify hand-held ultrasound probe connected to a Samsung Galaxy tablet with the Lumify app will help enhance participation in an ASA sponsored POCUS course.

\section{Disclosure}

Neither author has any competing interests or financial disclosures related to the devices described in this manuscript.

\section{References}

1. McCormick TJ, Miller EC, Chen R, Naik VN. Acquiring and maintaining point-of-care ultrasound (POCUS) competence for anesthesiologists. Can J Anaesth. 2018;65:427-436. doi:10.1007/ s12630-018-1049-7

2. Ramsingh D, Bronshteyn YS, Haskins S, Zimmerman J. Perioperative point-of-care ultrasound: from concept to application. Anesthesiology. 2020;132(4):908-916. doi:10.1097/ALN.0000000000003113

3. Meier I, Vogt AP, Meineri M, et al. Point-of-care ultrasound in the preoperative setting. Best Pract Res Clin Anaesthesiol. 2020;34 (2):315-324. doi:10.1016/j.bpa.2020.04.010

4. Meineri M, Arellano R, Bryson G, et al. Canadian recommendations for training and performance in basic perioperative point-of-care ultrasound: recommendations from a consensus of Canadian anesthesiology academic centres. Can J Anaesth. 2021;68(3):376-386. doi:10.1007/s12630-020-01867-2

5. Adler AC, Matisoff AJ, DiNardo JA, Miller-Hance WC. Point-ofcare ultrasound in pediatric anesthesia: perioperative considerations. Curr Opin Anaesthesiol. 2020;33(3):343-353. doi:10.1097/ACO.000 0000000000852

6. Adler AC, Chandrakantan A, Conlin FT. Perioperative point of care ultrasound in pediatric anesthesiology: a case series highlighting real-time intraoperative diagnosis and alteration of management augmenting physical examination. J Anesth. 2019;33(3):435-440. doi:10. 1007/s00540-019-02654-0

7. Baribeau Y, Sharkey A, Chaudhary O, et al. Handheld point-of-care ultrasound probes: the new generation of POCUS. J Cardiothorac Vasc Anesth. 2020;34(11):3139-3145. doi:10.1053/j.jvca.2020.07.004

8. Bronshteyn Y. Criteria for POCUS images. Available from: https:// education.asahq.org/totara/mod/giportfolio/view.php?id=24894. Accessed September 15, 2021.

9. ACGME program requirements for graduate medical education in anesthesiology. Available from: https://www.acgme.org/Portals/0/ PFAssets/ProgramRequirements/040Anesthesiology2018.pdf. Accessed September 15, 2021.

10. Deshpande R, Karnik R, Baer C, Kurup V. Resource utilization in implementation of a point of care ultrasound curriculum for resident training in anesthesiology. Yale J Biol Med. 2020;93:423-427.

11. Toscano M, Szlachetka K, Whaley N, Thornburg LL. Evaluating sensitivity and specificity of handheld point-of-care ultrasound testing for gynecologic pathology: a pilot study for use in low resource settings. BMC Med Imaging. 2020;20:121. doi:10.1186/s12880-02000518-8 
12. Li L, Yong RJ, Kaye AD, Urman RD. Perioperative Point of care ultrasound (POCUS) for anesthesiologists: an overview. Curr Pain Headache Rep. 2020;24(5):20. doi:10.1007/s11916-020-0847-0

13. Yamaguchi Y, Moharir A, Burrier C, Tobias JD. Point-of-care lung ultrasound to evaluate lung isolation during one-lung ventilation in children: a case report. Saudi J Anaesth. 2019;13:243-245. doi:10. 4103/sja.SJA_115_19
14. Lyman R, Yamaguchi Y, Moharir A, Tobias JD. Utility of point-ofcare ultrasound in the pediatric intensive care unit. Anaesth Pain Intensive Care. 2019;23:314-317.

15. Yamaguchi Y, Moharir A, Burrier C, et al. Point-of-care lung ultrasound to evaluate lung isolation during one-lung ventilation in children: narrative review. Med Dev Evid Res. 2020;13:385-389. doi:10. 2147/MDER.S265074

\section{Publish your work in this journal}

Advances in Medical Education and Practice is an international, peerreviewed, open access journal that aims to present and publish research on Medical Education covering medical, dental, nursing and allied health care professional education. The journal covers undergraduate education, postgraduate training and continuing medical education including emerging trends and innovative models linking education, research, and health care services. The manuscript management system is completely online and includes a very quick and fair peer-review system. Visit http://www.dovepress.com/testimonials.php to read real quotes from published authors. 